\title{
INGRESAR AL "ESTADO". MARCO DECISIONAL, ACTORES E INTERESES EN LOS PROCESOS DE INGRESO DE PERSONAL EN LA ADMINISTRACIÓN PÚBLICA CORRENTINA
}

\author{
VALERIA OJEDA \\ FACULTAD DE CIENCIAS ECONÓMICAS \\ UNIVERSIDAD NACIONAL DEL NORDESTE \\ IIGHI - CONICET
} ARGENTINA sino también por la suma de los pequeños empujones de cada trabajador honesto". Hellen Keller. 


\section{INTRODUCCIÓN}

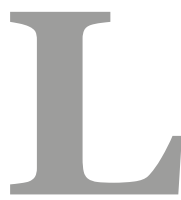

ste breve artículo reúne parte de los resultados de una tesis doctoral recientemente defendida para el Doctorado en Ciencias Sociales de la Universidad Nacional de Cuyo, denominada "Configuración de intereses en la Gestión de la Fuerza de Trabajo. Implicancias políticas e institucionales en la Administración Pública de Corrientes (1983-2012)”. El problema que aquí abordamos se circunscribe a las características que reúne el proceso de ingreso al empleo público, cuando el marco institucional para esta función se encuentra desactivado. Nuestro objetivo es entonces reconstruir la configuración del proceso de ingreso y conocer algunas de las estrategias que desarrollan los actores (trabajadores estatales-línea de decisión política) a fin de cumplimentar sus intereses. Asimismo revisar cómo se articulan las decisiones en torno a tales estrategias. Para la reconstrucción de estas formas y maneras de acceder al empleo (o de obtener personal) trabajamos a partir de entrevistas en profundidad realizadas a agentes estatales (mandos medios con responsabilidades de gestión de personal) de tres ministerios de la Administración Pública Provincial del nivel central, seleccionados a partir de un muestreo intencional. El tamaño de la muestra fue definido a través del criterio de saturación teórica.

Recordemos que el marco institucional que regula el ingreso al Estado provincial correntino se encuentra previsto en la Ley 4067, Ley de Servicio Civil de la Provincia. Esta ley, sancionada en 1986 durante el gobierno de José Antonio Romero Feris, considera de manera amplia los derechos y obligaciones tanto de los trabajadores como del Estado en su rol patronal: define también la naturaleza de las relaciones de trabajo establecidas, un sistema escalafonario, los procesos relativos a la movilidad (carrera) de los trabajadores, un régimen de licencias y franquicias, como aspectos más sobresalientes.

Sin embargo, como hemos podido comprobar en la tesis doctoral, gran parte del articulado de la Ley 4067 se encuentra discutido. Ya sea por ausencia de reglamentación, por solapamiento con otras leyes laborales provinciales (por ejemplo con la Ley de Sueldos y Remuneraciones 3801), o simplemente por desatención o incumplimiento, el sistema de concurso se encuentra desactivado. Esta inhabilitación responde a una serie de decisiones a nivel gubernamental, que se fueron replicando a lo largo de los casi 30 años que abordó nuestro trabajo. Es decir: los diferentes gobiernos consideraron que el acceso al empleo de los trabajadores se realice por fuera del sistema de concursos establecido por la Ley 4067 (excepto en tres ocasiones).

Tenemos por lo tanto diversos agentes que conocen las oportunidades que le brinda la configuración del proceso de ingreso, y que, en función de las mismas, activarán estrategias para lograr objetivos de su interés. Pero estos objetivos no serán iguales para todos ellos: se modificará en función del rol que cumplan. De esta forma se entretejerá una trama de intereses que calificará no solo la función de empleo sino también el resto de las funciones de GFT. 


\section{DESARROLLO}

Decíamos que desde 1986 está vigente un sistema de concursos que no se ha cumplimentado más que en tres oportunidades. Esto nos instala ante la necesidad de reconstruir el rol de los diversos gobiernos a lo largo de nuestro recorte temporal, incluso extendiéndolo, a fin de indagar en las prácticas que los mismos establecían alrededor del proceso de ingreso. Y es que cabe preguntarse respecto a la fortaleza del tejido institucional que soportó los procesos de gestión de los trabajadores dado que, siguiendo a North:

"Son las instituciones políticas y sociales de un país las que, cuando interactúan con condiciones económicas, determinan el potencial de oportunismo en y respecto de su administración pública, generando costos de transacción que hacen a las soluciones eficientes (más o menos) posibles, facilitan (más o menos) los intercambios a través del tiempo, posibilitan (más o menos) compromisos creíbles, y dotan de (más o menos) reputación (North, en Benedetti 1999: 18)”.

Asimismo es O'Donnell el que pone el acento en la complexión del tejido institucional de los Estados, al sostener que "los estados están entrelazados con sus sociedades respectivas de complejas y variadas maneras; esa inserción conduce a que los rasgos de cada uno de ellos y de cada sociedad tengan un enorme influjo sobre el tipo de democracia posible de consolidarse” (1993: 64).

Es posible transpolar esta noción al escenario de la administración pública: un diálogo entre conceptos de la Ciencia Política y las Ciencias Sociales del Trabajo puede ser fructífero en tanto la gestión de la fuerza de trabajo (o RRHH) no opera en el vacío. Los procesos de democratización de los espacios laborales dependen de manera muy estrecha de la complexión del tejido institucional. Son las características contextuales (políticas, organizacionales, culturales y sociales) las que irán otorgándole forma y sentido al mismo, en un juego iterado y dialéctico. Serán las instituciones informales las que darán cohesión y sentido a la gestión de la fuerza de trabajo. En mejores palabras, nuevamente recuperamos a O’Donnell:

“...cuando la concordancia es vaga o prácticamente inexistente, nos enfrentamos a la doble tarea de describir la conducta real y descubrir las reglas (generalmente informales) que rigen la conducta y las expectativas. Los actores son tan racionales en esos ambientes como lo son en los muy formalizados, pero no es posible delinear los perfiles de su racionalidad sin conocer las verdaderas reglas $-\mathrm{y}$ el conocimiento común de éstas- que ellos siguen (1996: 9)”.

Por tanto, nuestra indagación se desarrolló a partir de estas nociones. Y en este sentido 


\begin{tabular}{cc}
\hline & VALERIA OJEDA \\
$\mathbf{2 2 8}$ & INGRESAR AL “ESTADO”. MARCO DECISIONAL, ACTORES E INTERESES EN LOS PROCESOS... \\
Pags. $224-233$
\end{tabular}

de descubrir la conducta real, los encargados de personal a quienes entrevistamos, e incluso los informantes clave, pudieron dar cuenta de cuáles eran las costumbres y maneras que se establecían para conseguir personal, incluso de la década anterior a 1980. Las reseñaron a manera de referencia, como etapas en las cuales los niveles de ingresos se mantenían en niveles bajos y donde las decisiones eran sostenidas por un marco de formalidad. Consultábamos a un encargado de personal de 39 años de antigüedad respecto a si, en algún momento de su trayectoria laboral, los ingresos presentaron una mayor intensidad.

"Siempre fue así....te aclaro, yo no es que sea milico ni nada de eso pero la única época en que se respetó más o menos eso fue en esa época. ¿Ves? Eso es una cosa. Los concursos, eso que te hablé, se lo apuntaló en el gobierno militar. Cuando vino la democracia, no sé si ocurre en todas las provincias, yo te hablo desde mi experiencia personal que pasé todos los gobiernos. En esa época sí hubo concursos, había control sobre el personal, te daban el cargo que correspondía, yo en ese momento era pinche, pero el que estaba de jefe tenía el poder que le delegaban ellos, que es lo lógico".

Tanto informantes como entrevistados llamaron a los procesos de reclutamiento y selección previos a 1983 como "concursos", cuando en realidad no reunían las condiciones para considerarse como tales. Entre ellos indican períodos de contratos a prueba, presentaciones de carpetas de antecedentes y pruebas entre diferentes candidatos. Todos estos requisitos construían una imagen de respeto y formalidad sobre el proceso, cuando en realidad los ingresos terminaban siendo elecciones dirigidas por quienes tenían capacidad de agencia.

"En realidad en algunos casos era medio falsa la cosa, porque, en el sentido de que los funcionarios que estaban y que tenían que concursar el cargo por supuesto que hacían una serie de parodia, te imaginás vos, de antecedentes. Presentaban todos pero siempre aparecía un pariente, amigo, con el mejor antecedente! Pero...de alguna manera había una formalidad”.2.

La atención a la formalidad era, por lo tanto, una característica que reunían estos procesos de ingreso a la Administración del Estado. El período que señalan los informantes se ubica entre 1972 y 1983, y coinciden con etapas de gobierno peronista (Julio Romero) y de gobierno de facto. Fueron reiteradas las emisiones de nuestros entrevistados a aquella etapa anterior a 1983 como una época de menos compromisos, "porque si por algo se caracterizaron los milicos, era que no tenían compromiso con nadie"3. Sin embargo la existencia de compromisos excedía la naturaleza

\footnotetext{
${ }^{1}$ Entrevistado 10.

2 Informante B.

${ }^{3}$ Informante $\mathrm{C}$. 
del gobierno de turno. Gobiernos militares o civiles generaban redes de conocimiento, de cercanía, incluso de lealtad, que se expresaban también sobre la función de empleo:

"Y entonces, siempre entre conversaciones así, mi hermano me dice 'mirá ahí hay una vacante en la legislatura ahí, para estar ahí en atención al público y que se yo..... Me dice: 'es más que tu jornalizado'. iEncima era de planta! 'Eh... ¿̇querés? Porque yo estoy manejando el tema de los antecedentes, que se yo, se está recibiendo ahí, por supuesto que la palabra la tiene el subsecretario porque es el que determina después el entre'. Entonces agarro y le digo 'si pue'! todo ahí pue”.

Notemos que en esta expresión se suman al proceso de ingreso otros actores. Ya no es sólo una relación directa en donde interviene el Estado (representantes del Estado con poder de decisión para determinar el ingreso) y el postulante. Aquí identificamos un facilitador, una persona que, si bien no cuenta con "la palabra", sí conoce los mecanismos para acercar a las partes. En este caso el "hermano" del entrevistado operó mediando el proceso de ingreso.

Para continuar revisando cómo se articuló el proceso de empleo a lo largo de las décadas que abarca esta tesis nos es preciso atender, brevemente, a las condiciones políticas e institucionales que enmarcaron el pasaje del gobierno militar al democrático. De manera clara fueron nuestros entrevistados quienes retomaron estas condiciones como sustrato explicativo de la permanencia de este tipo de prácticas para ingresar personal en la Administración del Estado.

¿Cuánto de cambio hubo entre la salida del gobierno de facto y el reinicio de la democracia? ¿Por qué reviste interés considerar las condiciones políticas e institucionales? Un logro importante a nivel de información que nos dejó el relevamiento realizado a través de las entrevistas y consultas a informantes clave ha sido conectar la etapa anterior a 1983 a la problematización planteada en este trabajo. Al pensar el recorte temporal iniciándolo desde la recuperación de la Democracia entendíamos que esta reconquista institucional plantearía un marco diferenciado, al recobrarse las garantías constitucionales y una reactivación del derecho administrativo. Sin embargo otros aspectos son los que concurrieron para determinar las características de la Administración Pública como espacio social y laboral.

La relación establecida entre los núcleos de poder político y las jerarquías militares condicionaron fuertemente el escenario laboral en el empleo público. Lejos de pensarse como sectores antagónicos, una porción del arco político correntino permaneció relacionado de manera directa e incluso con funciones dentro del gobierno militar. A partir de ese vínculo se fue construyendo una base de poder que se trasladó, una vez recuperada la democracia, a los diversos estamentos del Estado. Nuestros informantes clave nos señalaron que fueron los sectores conservadores, representados por los partidos Liberal y Autonomista ("El Pacto") quienes, entre 1976 y 1983 ocuparon espacios de decisión dentro de la estructura del Estado, incluso lugares

${ }^{4}$ Informante B. 
de importancia tales como secretarías y/o ministerios.

"Un dato, para entender de la complicidad de todo el conservadorismo, y fundamentalmente del PAL, es que Leconte ${ }^{5}$ fue intendente de la dictadura, fue intendente de la ciudad de Corrientes en la dictadura. Y el Secretario general de la Intendencia de la Municipalidad, Braillard Poccard 6 . Era un pibe”.

“Llegan las elecciones, gana Alfonsín, y acá gana Pocho Romero Feris ${ }^{8}$, el pacto, o sea la continuidad de la dictadura digamos, los conservadores. Porque ellos, con Pita (gobernador militar) estaban como si estuviera gobernando. Tenían divididos los ministerios, por ejemplo: el ministerio de gobierno era autonomista, se dividían la estructura, vialidad era liberal, economía era esto, así, así como funcionaba, así hizo Pocho. En realidad es porque ellos ya estaban gobernando y entonces la mayoría de los puestos que habían ingresado en la Administración Pública en la etapa de la dictadura eran del pacto".

De esta forma, la administración del Estado Provincial a partir de la recuperación de la democracia parte de un escenario pre-formado durante el proceso militar. En tal sentido la incorporación de agentes a las estructuras del Estado a partir de procesos de ingreso aparentemente formalizados fue una situación característica del período inmediato anterior al 83. Asimismo los ingresos estaban apoyados en redes de compromiso que se articulaban no sólo con el poder militar sino también de la interrelación de éstos con el sector político conservador de Corrientes.

Entonces tenemos, al iniciar el recorte temporal (1983), una estructura administrativa del Estado que ya había sido intervenida por un componente político de relevancia, y que luego, recuperada la Democracia, obtuvo de manera legítima la potestad de decidir sobre la misma. Las referencias cualitativas que rescatamos respecto de los procesos de ingreso durante el gobierno de José Antonio "Pocho" Romero Feris lo marcan como el gobierno a partir del cual se habilitaron ingresos de manera masiva.

"Sin ninguna duda el inicio de la democracia. El peor daño que le hizo Pocho a la Administración Pública, en ese ingreso masivo, en esa necesidad de, en una provincia como la nuestra donde el trabajo principal es el servicio a la Administración Pública, terminó deformando absolutamente la administración. De ahí surge la necesidad de la 4067, y él, capaz pensando bueno, vamos a conformar-

\footnotetext{
${ }^{5}$ Ricardo Leconte, gobernador de la Provincia de Corrientes entre 1987 y 1991, de extracción Liberal.

${ }^{6}$ Accedió a la gobernación de la Provincia en diciembre de 1997. Inicialmente Liberal luego formó las filas del Partido Nuevo, de Raúl "Tato" Romero Feris. A través de ese partido llegó a la gobernación. Fue destituido por Juicio Político en el año 1999.

${ }^{7}$ Informante F.

${ }^{8}$ Gobernador de la Provincia entre 1983 y 1987, de extracción Autonomista.

${ }^{9}$ Informante B.
} 
le con la ley' creó....ese inicio nunca se pudo corregir, nunca"ı.

Sin embargo las estadísticas elaboradas también para nuestro trabajo de tesis ${ }^{11}$, generadas a partir de los datos históricos de liquidación de haberes facilitados por el SISPER, lejos están de marcarla como la gestión que más personal ingresó durante sus años en el gobierno. Nuestro entrevistado señala que "ese inicio nunca se pudo corregir", haciendo referencia a que, es a partir de la recuperación de la democracia cuando se habilita la intervención política como la variable más importante en el acceso al empleo en el Estado. Lo particular de esta etapa es que, a pesar de que los procesos de ingreso estuvieran mediados por decisiones sesgadas, y/o lejanas a criterios administrativos (tales como los que califican el proceso de empleo) de igual manera se intentó dotar de sistematicidad a la GFT estableciendo un marco normativo inicial con la sanción de la Ley 4067 y su posterior reglamentación.

\section{CONCLUSIONES}

Tal como lo hemos mencionado en esta ponencia breve, establecer en este caso, caracteres definitorios del proceso de empleo por cada gestión de gobierno resultará en un ejercicio vano. A partir de nuestros acercamientos al fenómeno de la configuración de intereses sobre la Gestión de la Fuerza de Trabajo en la Administración del Estado Provincial Correntino pudimos ir observando que hay un aprendizaje que trasciende cada gestión de gobierno y que por lo tanto se ancla a nivel de la misma organización. Más ello no nos limita el considerar aquellos aspectos salientes o destacables que hayan surgido de las voces de nuestros entrevistados y que refieran a determinadas gestiones de gobierno. Por ello continuaremos el análisis no desde una perspectiva cronológica (lo cual nos llevaría a abordar la gestión de Ricardo Leconte) sino que lo realizaremos en función de este determinante metodológico.

Si retomamos el foco (revisar cómo se articulan las decisiones en torno al proceso de empleo, quiénes son sus actores y bajo qué intereses actúan), nos resulta pertinente traer al análisis las percepciones de los encargados de personal respecto de los niveles de ingreso en cada gobierno desde 1983 al 2012. Para acercarnos a estas reflexiones proveíamos a nuestros entrevistados de una planilla que ilustraba las cantidades de trabajadores ingresados en cada una de las ocho gestiones de gobierno y procedíamos a observarlas de manera conjunta. De este ejercicio pudimos rescatar algunas categorías importantes.

\footnotetext{
${ }^{10}$ Informante C

${ }^{11}$ Puestos creados por gestión: J.A. Romero Feris: 10321. R. Leconte: 6815. Intervención 91-92: 6488. R.R. Romero Feris: 14292.

P. Braillard Poccard: 9126. Intervención 00-01: 6974. R.Colombi (I): 26467. A. Colombi: 40693. R.Colombi: 29598.
} 
La primera de ellas está en relación a cierto "cuidado" que tienen los gobiernos, y sus principales referentes, de que los movimientos de personal sean progresivos, o por lo menos que no llamen la atención. En cierta forma se trata de conservar la discreción y proceder a tomar personal a partir de las estrategias que hemos venido relatando.

(La entrevistada observa la planilla de movimientos de personal por gestión. Compara el nivel de ingresos entre las gestiones de Ricardo y Arturo Colombi, y la de José A. Romero Feris)

"Totalmente, sin ninguna duda. Por lo que yo vi durante todos estos años. Acá no te discuto que hubo (señala gestión J.A. Romero Feris) iOjo! pero era más solapado más cuidado, más no sé qué. O sea, era menos. Esto ya es alevoso (señala gestión Arturo Colombi)”.

Al comparar diversas gestiones y sus niveles de ingreso, nuestros entrevistados señalaban que en algunas eso era "alevoso"13 y que "no les importaba"14. Otros consideraban que estas desproporciones e ingresos tan desmesurados se debían a una falla en la cadena de decisiones, las cuales no pasaban estrictamente por el responsable de gobierno sino que se perdían en una multiplicidad de niveles. Esto lo determinamos trayendo a colación la imagen construida por la opinión pública respecto a que, en la gestión de Raúl “Tato” Romero Feris se produjeron los ingresos más numerosos.

"Es que todos dicen lo mismo pero no, no fue así. Esta gestión, cada uno de estos hombres que están acá arriba (señala a R. Colombi y A. Colombi) ni saben, ni saben. En cambio estos que estaban acá sabían (señala R. Romero Feris, P. Braillard Poccard). Para mi tienen una venda en el ojo. Porque la persona que le lleva el nombre, la propuesta...es toda una cadenita, y sobre esa cadenita no hay control"15.

Por lo tanto la presencia de un liderazgo fuerte, centralizador de las decisiones, es la característica que remite a nuestros entrevistados a considerar determinante para el control de los niveles de ingreso y por lo tanto mantener esta “discreción” a la que aludíamos párrafos más arriba.

Finalmente, nos resta señalar que los encargados de personal rescataron que, durante las Intervenciones Federales, los procesos de ingreso presentaron corrimientos o formas "distintivas" a las habituales. El componente decisional en estos períodos no se establecía a partir de compromisos sino que los intereses que operaban sobre tales decisiones se vinculaban a la encomienda recibida y la naturaleza de las funciones de una Intervención Federal (sanear el Estado, promover la reorganización institucional).

\footnotetext{
12 Informante $\mathrm{H}$.

${ }^{13}$ Entrevistado 3.

${ }^{14}$ Entrevistado 8.

${ }^{15}$ Entrevistado 12.
} 
"Una de las etapas que siempre se manejaron mejor fue durante las intervenciones. Salvo que ellos hayan traído a alguien, pero generalmente no trajeron nunca gente, o si no estaban de nn ntro del grupo de autoridades. No tenían nada que ver con planta o contrato todo dejaban que manejemos nosotros" ${ }^{\prime 6}$.

En el mismo sentido, la ausencia de cercanía o amistad con las redes establecidas dentro de las estructuras de gobierno no permitieron a los funcionarios de las Intervenciones Federales flexibilizar los vínculos, "enchamigarse"17, en palabras de los mismos trabajadores. De esta manera también la falta de lazos de familiaridad y conocimiento personal condicionaron las decisiones en cuanto a ingresos de nuevos trabajadores.

\section{REFERENCIAS BIBLIOGRÁFICAS}

Benedetti, P. (1999). Aspectos institucionales de la administración pública. Implicancias para la argentina. Fundación Gobierno y Sociedad. Disponible en: faculty.udesa.edu.ar/tommasi/ cedi/dts/dt22.PDF

O’Donnell, G. (1993). Estado, democratización y ciudadanía. Nueva Sociedad № ${ }^{128}$. 62-87.

(1996) Ilusiones sobre la consolidación. Nueva Sociedad Nro. 144. Julio - Agosto 1996, 70-89. Buenos Aires.

\section{CURRICULUM VITAE}

\section{Valeria Ojeda}

Doctora en Ciencias Sociales, con mención en Administración Pública y Ciencia Política por la Universidad Nacional de Cuyo. Es docente regular de la Licenciatura en Relaciones Laborales de la FCE y becaria posdoctoral CONICET-UNNE, desarrollando actividades de investigación en el IIGHI (CONICET-UNNE).

leryval@hotmail.com

\footnotetext{
${ }^{16}$ Entrevistado 8.

${ }^{17}$ El término "enchamigarse" es de uso habitual en el habla de los correntinos. Podríamos intentar realizar una definición del término como "la condición de adquirir familiaridad a partir de la flexibilización del vínculo o relación establecida entre dos personas con diferentes posiciones relativas. "Hacerse amigo de".
} 